Max-Planck-Institut für demografische Forschung

Max Planck Institute for Demographic Research

Konrad-Zuse-Strasse 1 < D- 18057 Rostock < Germany < Tel +49 (0) 3812081 - 0 < Fax +49 (0) 3812081 - 202 < w w w.demogr.mpg.de

M PIDR W orking Paper WP 2017-017 I October 2017

\title{
Expanding the Markov chain tool box: Distributions of occupation times and w aiting times
}

Christian Dudel I dudel@demogr.mpg.de

\footnotetext{
This working paper has been approved for release by: Sebastian Klüsener (kluesener@ demogr.mpg.de), Deputy Head of the Laboratory of Fertility and W ell-Being.

(c) Copyright is held by the authors.

Working papers of the Max Planck Institute for Demographic Research receive only limited review. View s or opinions expressed in working papers are attributable to the authors and do not necessarily reflect those of the Institute.
} 


\title{
Expanding the Markov chain tool box: Distributions of occupation times and waiting times
}

\author{
Christian Dudel* \\ Max Planck Institute for Demographic Research
}

\begin{abstract}
Markov models are important tools for quantitative social research. In this paper, new methods for discrete Markov chains are presented. These methods allow us to calculate the distribution of the occupation time in a subset of the state space, the distribution of the waiting time to first entry into a subset of the state space, and the distribution of the waiting time to final exit from a subset of the state space. To demonstrate the usefulness of these methods, we apply them to working life tables for Spanish males to assess how the recent financial crisis affected the length of working life. The results show that the duration of working life decreased considerably, a pattern that can largely be explained by later entry to and earlier exit from the labor market. The findings also indicate that inequality in the length of working life increased.
\end{abstract}

\footnotetext{
*Contact: Max Planck Institute for Demographic Research, Konrad-Zuse-Str. 1, 18057 Rostock, Germany; email: dudel@demogr.mpg.de; phone: +49 381 2081221; fax: +49 3812081521
} 


\section{Introduction}

Markov models are important tools for quantitative social research. Classical applications include the modeling of social and occupational mobility (Prais, 1955; Hodge, 1966) and migration (Rogers, 1975). Recent examples are the analysis of transitions between family states (e.g., Willekens et al., 1982; Schoen et al., 2007), the estimation of healthy life expectancy (e.g., Rogers et al., 1990; Crimmins et al., 1996; Lubitz et al., 2003; Karas Montez and Hayward, 2014), and applications in criminology (e.g., Stander et al., 1989; Schmertmann et al., 1998).

The popularity of Markov models stems from the ease with which they can be applied: given a set of probabilities or rates describing the transitions between some states, a rich set of methods can be used to derive quantities like the average time spent in a state (Kemeny and Snell, 1971; Iosifescu, 1980; Taylor and Karlin, 1984). Most of these methods for Markov models were established well before the 1970s. Despite their usefulness, methods for Markov models have received little attention in recent years, in contrast to other aspects like model estimation (e.g., van den Hout and Matthews, 2009; Wolf and Gill, 2009; Schoen, 2016).

This paper expands the tool box for Markov models. We combine earlier work by Sericola (2000) and Skoog and Ciecka (2010), and develop three methods that can be applied to gain deeper insight into the dynamics captured by these models: one method for occupation times and two methods for waiting times. Starting from a discrete-time, finite state space, homogeneous Markov chain, and considering a finite interval of time, the method for occupation times allows us to calculate the distribution of time units spent in any arbitrary subset of the state space. The methods for waiting times presented in this paper allow us to assess the timing of events in Markov chains. The first of these methods can be used to calculate the distribution of the waiting time to first entry into a subset of the state space, while the second method gives the distribution of the waiting time to the final exit from a subset of states, potentially through another specific subset of states.

To better understand what can be achieved with the methods presented here, assume that we are interested in the migration of individuals between several regions; say, regions $A, B$, and $C$. More specifically, we are interested in the migration of individuals from region $C$ to the other regions. Moreover, assume that regions $A$ and $B$ are in some sense similar. For modeling, we use a Markov chain in which the regions are the states of the chain. Applying the method for occupation times presented in this paper, we can then assess how much time is spent in either region $A$ or $B$; i.e., in a subset of the state space; from this result, we can calculate the average time, the median time, the variance, etc. Note that knowing both the distribution of time spent in $A$ and the distribution of time spent in $B$ is not equivalent to knowing the distribution of time spent in either $A$ or $B$; but that all of these distributions might be of interest. Applying the methods for timing of events, we can calculate the distribution of the waiting time until an individual from $C$ migrates to $A$ or $B$ or to either of them, conditional on migrating. The method for calculating the time to final exit allows for multiple transitions between $C$ and $A$ and $B$; that is, individuals can migrate repeatedly between $C$ and $A$ and $B$. The method presented here could be used to calculate the distribution of the time until 
individuals finally fully relocate from $C$ to either $A$ or $B$, or the distribution of the time until individuals from $C$ permanently and finally return to $C$ after migrating to $A$ and $B$.

The methods presented in this paper contribute to the literature in several ways. While most other Markov chain methods yield averages, the methods presented here yield complete distributions. Any statistic of interest can be calculated based on these distributions, such as, for example, measures of inequality. Looking not just at specific single states, as Markov chain methods usually do, but at subsets of states, is especially useful when applying complex models with many states. Assessing the timing of events like first entry or final exit is often of prime interest to the researcher, as doing so enhances our understanding of the social process modeled through a Markov chain. Up to now, methods that allow us to assess the timing of events have been limited. To ensure that these methods are readily usable, $\mathrm{R}$ code is supplied with this paper, and can be accessed online.

To provide an example of how the methods presented in this paper can be used, we apply them to period working life tables for Spanish males. Working life tables describe transitions between labor market states. For instance, the state space could consist of the states "active" (either employed or unemployed and looking for work), "inactive" (out of the labor market), "retired", and the absorbing state "dead". Given estimates of transition probabilities, it is standard practice to calculate the expected lifetime spent in each of the states, including the expected lifetime spent in the "active" state, which is of prime policy interest, and is called working life expectancy (Loichinger and Weber, 2016). In the literature, working life tables have been reported primarily for the United States (Warner et al., 2010; Skoog and Ciecka, 2010; Millimet et al., 2010, 2003; Hayward and Richter, 1998; Hayward et al., 1996; Hayward and Grady, 1990; Smith, 1986), but working life tables are also available for Finland (Leinonen et al., 2016; Nurminen et al., 2005), the United Kingdom (Haberman and Bloomfield, 1990; Butt et al., 2008), the Netherlands (Liefbroer and Henkens, 1999), and Denmark (Hoem, 1977; Willekens, 1980).

In this paper, working life tables for Spanish males are calculated using Spanish social security data for the years 2004 to 2013. During this period, Spain was hit hard by the financial crisis. Unemployment increased dramatically (Jimeno and Santos, 2014), while working life expectancy decreased (Dudel et al., 2016). The highly segregated structure of the Spanish labor market (Bentolila et al., 2012a) might imply that the impact of the recession was heterogeneous. To assess this potential heterogeneity of the impact of the crisis, we calculate the distribution of the lifetime spent in the "active" state, and assess how the recession affected the quartiles of this distribution. We then use the interquartile range to assess for the first time the inequality in the length of working life. Furthermore, it is not clear to what degree the decrease in working life expectancy is due to a compression of working life (i.e., to later entry into and earlier exit from the labor force), or to unstable career trajectories (i.e., phases of withdrawal from the labor market during working life). There is evidence for both effects (Dolado et al., 2013; Congregado et al., 2011). Using the methods presented in this paper, we calculate the first time an individual enters the labor market and the final time the individual leaves the labor market; thus, we are able to disentangle the effects of later entry and earlier exit. Our methods are especially well suited for this, as they take into account that leaving the 


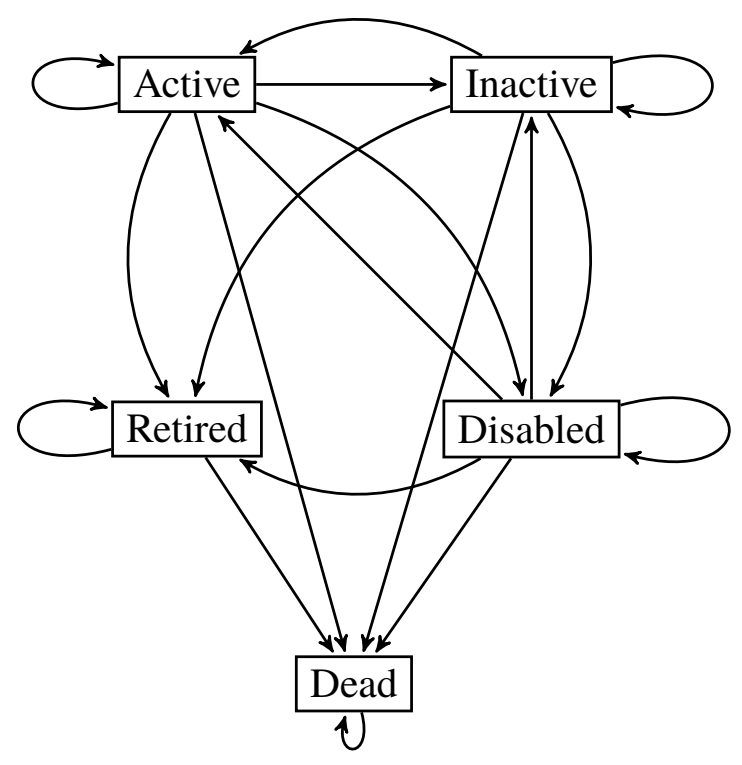

Figure 1: Simplified state space of the working life table ignoring age.

labor market can happen through different pathways (e.g., a period of unemployment before retirement) and that individuals might return to work.

The remainder of this paper is structured as follows. Preliminaries and notation are introduced in section 2. The calculation of the distribution of the time spent in a subset of states is introduced in section 3, while the timing of events - i.e., first entry and final exit - is discussed in section 4. The empirical application is presented in section 5. Section 6 concludes.

\section{Notation and preliminaries}

\subsection{The state space}

Let $\mathcal{S}=\left\{s_{1}, s_{2}, \ldots\right\}$ denote the finite state space consisting of states $s_{j}$. For the empirical application presented later in section 5, the state space will consist of the labor force states "active", "inactive", "retired", and "disabled", all combined with age, with 15 being youngest and 99 years being the oldest age considered. For instance, there are states labeled as "aged 15 and inactive", "aged 16 and inactive", and so on, until "aged 99 and inactive". The state space also includes the absorbing state "dead". Transitions are only allowed between states of consecutive age, e.g., a transition from "aged 20 and employed" to "aged 21 and inactive", with the exception of "dead", which can always be reached. This means that aging and survival and transitions between labor force states are modeled jointly. The retired state can be left only through death, as Spaniards rarely return to work after retirement. A simplified version of the state space ignoring age is shown in figure 1.

Let $\mathcal{A}$ and $\mathcal{B}$ be a partition of the state space; i.e., some of the states are collected in $\mathcal{A}$, while the remaining states are in $\mathcal{B}$. For example, for some of the calculations in 
the empirical application, $\mathcal{A}$ will be defined as

$\mathcal{A}=\{$ aged 15 and active, aged 16 and active, .., aged 99 and active $\}$,

i.e., $\mathcal{A}$ includes all "active" states, while $\mathcal{B}$ includes all "inactive", "retired" and "disabled" states as well as "dead".

\subsection{Counting conventions and transitions}

$D$ will denote the random variable that captures the time spent in subset $\mathcal{A}$; e.g., the time spent in the "active" labor force state. $V$ denotes the random variable that captures the time to the first visit of $\mathcal{A}$, and $E$ denotes the time to the last exit of $\mathcal{A}$; e.g., the time to the final exit from the active state. $D=0$ can be interpreted to mean that no time has been spent in $\mathcal{A}$. For $V$, it is assumed that $V=0$ means that the first entry already has occurred. If the first entry never happens, it is assumed that $V=\infty . E=0$ means that the final exit either has already has occurred or will never occur.

Time $t$ is assumed to be discrete; i.e., $t=0,1,2, \ldots{ }^{1}$ All of the methods presented in this paper are over a finite interval of time, starting from time 0 and running to some other time $t^{\prime}$. Transitions are assumed to occur mid-interval, as is common in many social science applications (e.g., Skoog and Ciecka, 2010). ${ }^{2}$ The variables can thus take on the following values:

$$
\begin{aligned}
& D=0,0.5,1,1.5,2,2.5, \ldots \\
& V=0,0.5,1.5,2.5,3.5, \ldots \\
& E=0,0.5,1.5,2.5,3.5, \ldots
\end{aligned}
$$

An example that illustrates the reasoning behind this approach is depicted below. The example shows the working trajectory of a single individual. $Z_{t}$ captures the state the individual is in at time $t$, where $a$ denotes that the individual is active in the labor market, $o$ denotes that the individual is inactive or is out of the labor force, and $r$ stands for retired:

$$
\begin{array}{ccccc}
Z_{0}=a & Z_{1}=a & Z_{2}=o & Z_{3}=r & Z_{4}=r \\
t=0 & t=1 & t=2 & t=3 & t=4
\end{array}
$$

The process starts at time $t=0$, when the individual is employed. At $t=1$, the individual is still employed; at $t=2$, she is retired; and so on. If $\mathcal{A}=\{r\}$, and the researcher's interest lies in determining the time spent in retirement, $D_{t}$ develops as

\footnotetext{
${ }^{1}$ Note that this does not restrict the applicability of the methods presented in this paper to discrete models. Given estimates of the transition intensities of a continuous model, discrete transition probabilities can easily be calculated (e.g., Rogers and Ledent, 1976).

${ }^{2}$ The results presented in this paper can easily be adapted to different counting conventions. For instance, if transitions are assumed to occur at the end of the interval, formulas for the probability of waiting 0.5 time units can be used for the probability of waiting one time unit.
} 
follows. $D_{0}$ equals zero, as the process has just started and no time has passed. $D_{1}$ still equals zero, as the individual has not yet spent time in retirement. The same holds for $D_{2}$. Between $t=2$ and $t=3$, the individual moves from being out of the labor force to retirement. As the transition is assumed to occur mid-interval, $D_{3}$ equals 0.5. $D_{4}$ equals 1.5 , because the individual stayed in retirement. The time to the first entry and the time to the last exit follow similar rules. $V_{0}$ equals $2.5, V_{1}$ equals 1.5 , and $V_{2}$ is 0.5 , while the values of $E_{t}$ will depend on how the trajectory develops after $t=4$.

\subsection{Transition probabilities}

Transition probabilities $\operatorname{Pr}\left(Z_{t+1}=s_{j} \mid Z_{t}=s_{i}\right)$ capture the probability that an individual in state $s_{i}$ at time $t$ will be in state $s_{j}$ at time $t+1 . p_{i j}$ is used as a shorthand for $\operatorname{Pr}\left(Z_{t+1}=s_{j} \mid Z_{t}=s_{i}\right)$. The transition probabilities only depend on the present state of the process at $t$ and not on the history at $t-1, t-2$, and so on. For this reason, the Markov chain has the Markov property, and is memoryless. It is also assumed that the Markov chain is homogeneous, meaning that the transition probabilities do not depend on $t$, and thus do not change over time; and that the Markov chain is absorbing, which implies that there is at least one state that will eventually be reached with probability one and that cannot be left once reached. The latter assumption simplifies the application of the Markov methods presented here, even though it is not strictly necessary.

$\mathbf{P}=\left[p_{i j}\right]$ denotes the transition matrix of the Markov chain that includes all transition probabilities. Using this transition matrix, several quantities can be calculated (for a general overview, see Kemeny and Snell, 1971). The expected time spent in state $s_{j}$ starting from state $s_{i}, n_{i j}$, is given by

$$
\mathbf{N}=\left(\mathbf{I}_{s}-\mathbf{U}\right)^{-1}
$$

where $\mathbf{U}$ is a transition matrix that does not include absorbing states, and $\mathbf{I}_{s}$ is an identity matrix of dimension $s \times s$, where $s$ is the number of non-absorbing (transient) states. Note that this equation follows a different counting convention, and assumes that transitions occur at the end of intervals. Because of this assumption, $n_{i i}$ is overestimated by 0.5 , and needs to be adjusted accordingly. The row sums of $\mathbf{N}$ minus 0.5 give the life expectancy conditional on starting in state $s_{i}$ (Caswell, 2001).

\section{Time spent in a subset of states}

Previously published studies that provided approaches for calculating the distribution of the occupation time include Iosifescu (1980), Sericola (2000), and Skoog and Ciecka (2002, 2010). Skoog and Ciecka $(2002,2010)$ described an approach for a model restricted to two non-absorbing states. Using different counting conventions, Iosifescu (1980) showed how the distribution of the time spent in one specific state can be derived. The formulas presented in this paper are more general, as they assess the time spent in a subset of states, and they allow for an arbitrary number of (non-)absorbing states. They are modified versions of the formulas given by Sericola (2000), and they are closely related to first-step analysis, one of the basic tools of Markov chain analysis (e.g., Taylor 
and Karlin, 1984). The formulas are modified such that they follow the assumptions regarding mid-interval transitions outlined in the previous section, whereas Sericola (2000) assumed that transitions occur at the end of intervals.

The starting point is the distribution $\operatorname{Pr}\left(D_{t}=d \mid Z_{0}=s_{i}\right)$; i.e., the distribution of the time spent in a subset of states $\mathcal{A}$, conditional on starting in state $s_{i}$. Conditioning on the starting state $s_{i}$ is common for Markov chain methods. If the researcher is interested in the unconditional distribution $\operatorname{Pr}\left(D_{t}=d\right)$, it can be derived by combining $\operatorname{Pr}\left(D_{t}=d \mid Z_{0}=s_{i}\right)$ with a distribution of $Z_{0}$, whereby the latter can be either estimated empirically or determined through subject knowledge about the structure of the process.

$\operatorname{Pr}\left(D_{t}=d \mid Z_{0}=s_{i}\right)$ is given by $\operatorname{Pr}\left(D_{t} \leq d \mid Z_{0}=s_{i}\right)-\operatorname{Pr}\left(D_{t} \leq d-1 \mid Z_{0}=s_{i}\right)$. $\operatorname{Pr}\left(D_{t} \leq d \mid Z_{0}=s_{i}\right)$ can be obtained from the row sums of $\mathbf{D}(t, d)$, where $\mathbf{D}(t, d)=$ $\left[\operatorname{Pr}\left(D_{t} \leq d, Z_{t}=s_{j} \mid Z_{0}=s_{i}\right)\right]$ captures the probability that conditional on starting in state $s_{i}$ the process is in state $s_{j}$ at time $t$, and the time spent in $\mathcal{A}$ is at most $d$ time units. To calculate $\mathbf{D}(t, d), \mathbf{P}$ is partitioned with respect to $\mathcal{A}$ and $\mathcal{B}$,

$$
\mathbf{P}=\left(\begin{array}{ll}
\mathbf{P}_{\mathcal{A}} & \mathbf{P}_{\mathcal{A B}} \\
\mathbf{P}_{\mathcal{B} \mathcal{A}} & \mathbf{P}_{\mathcal{B}}
\end{array}\right)
$$

where $\mathbf{P}_{\mathcal{A}}$ includes the transition probabilities $p_{i j}$, where both $s_{i}$ and $s_{j}$ are in $\mathcal{A}, \mathbf{P}_{\mathcal{A B}}$ includes the transition probabilities $p_{i j}$ for which $s_{i}$ is in $\mathcal{A}$ and $s_{j}$ is in $\mathcal{B}$, and so on.

$\mathbf{D}(t, d)$ is then calculated recursively. For $t=0$, the initial conditions are given by

$$
\mathbf{D}(t, 0)=\mathbf{I}_{m}
$$

where $\mathbf{I}_{m}$ is a $m \times m$ identity matrix and $m$ is the total number of states. This means that no time has been spent in subset $\mathcal{A}$ before the process starts. For $t>0$ initial conditions are

$$
\mathbf{D}(t, 0)=\left(\begin{array}{cc}
\mathbf{0} & \mathbf{0} \\
\mathbf{0} & \mathbf{P}_{\mathcal{B}}^{t}
\end{array}\right)
$$

and

$$
\mathbf{D}(t, d)=\mathbf{P}^{t} \quad \text { for } d>t .
$$

Condition (4) states that no time spent in $\mathcal{A}$ up to time $t$ requires not leaving subset $\mathcal{B}$. Condition (5) means that after $t$ time units, the time spent in $\mathcal{A}$ cannot be greater than $t$, and has to be equal to or less than $t$.

Using these initial conditions and the decomposition of $\mathbf{P}, \mathbf{D}(t, d)$ is computed as

$$
\begin{aligned}
\mathbf{D}(t, d) & =\left(\begin{array}{ll}
\mathbf{P}_{\mathcal{A}} & \mathbf{0} \\
\mathbf{0} & \mathbf{0}
\end{array}\right) \mathbf{D}(t-1, d-1)+\left(\begin{array}{ll}
\mathbf{0} & \mathbf{0} \\
\mathbf{0} & \mathbf{P}_{\mathcal{B}}
\end{array}\right) \mathbf{D}(t-1, d) \\
& +\left(\begin{array}{ll}
\mathbf{0} & \mathbf{P}_{\mathcal{A B}} \\
\mathbf{P}_{\mathcal{B} \mathcal{A}} & \mathbf{0}
\end{array}\right) \mathbf{D}(t-1, d-0.5)
\end{aligned}
$$

for $d=0.5,1,1.5,2, \ldots$ and $t>0$. The proof follows directly from Sericola (2000) and exploits homogeneity and the Markov property. More specifically, $\operatorname{Pr}\left(D_{t} \leq d, Z_{t}=\right.$ 
$\left.s_{j} \mid Z_{0}=s_{i}\right)$ can be decomposed into $\sum_{s_{k} \in \mathcal{S}} \operatorname{Pr}\left(D_{t} \leq d, Z_{t}=s_{j} \mid Z_{1}=s_{k}\right) \operatorname{Pr}\left(Z_{1}=s_{k} \mid Z_{0}=\right.$ $\left.s_{i}\right)$ which equals $\sum_{s_{k} \in \mathcal{S}} \operatorname{Pr}\left(D_{t-1} \leq d-\mathrm{I}\left(s_{i}, s_{k}\right), Z_{t-1}=s_{j} \mid Z_{0}=s_{k}\right) p_{i k}$, where

$$
\mathrm{I}\left(s_{i}, s_{k}\right)=\left\{\begin{array}{lll}
0 & \text { if } & s_{i}, s_{k} \in \mathcal{B} \\
0.5 & \text { if } & s_{i} \in \mathcal{A}, s_{k} \in \mathcal{B} \\
0.5 & \text { if } & s_{i} \in \mathcal{B}, s_{k} \in \mathcal{A} \\
1 & \text { if } & s_{i}, s_{k} \in \mathcal{A}
\end{array} .\right.
$$

Recursion (6) restates these equations in matrix notation.

\section{Timing of events in Markov models}

\subsection{Waiting time to first entry}

The existing body of work on methods for calculating the time to first entry (sometimes called first passage) is small. Grinstead and Snell (1997) presented a method for calculating the average time needed to reach state $s_{i}$ from state $s_{j}$, but it is only applicable to ergodic Markov chains. The approach presented here does not assume ergodicity. Moreover, it guarantees that the entry really is the first. For this reason, the formulas given below differ somewhat from those for the time spent in a subset of states. For the time spent in a state, it is sufficient to consider the history of the process; i.e., how many times a subset of states has been visited up to time $t$. For the time to first entry, it is necessary to consider both what happened before $t$ - i.e., whether the subset has been already entered - and how the process develops after $t$-i.e., when the subset will be visited for the first time (if it has not been visited before).

Let $\operatorname{Pr}\left(V_{t}=v \mid Z_{0}=s_{i}\right)$ denote the distribution of the time needed to reach a state in $\mathcal{A}$ starting from state $s_{i}$. The formulas given below allow us to calculate $\operatorname{Pr}\left(V_{t} \geq v, Z_{t}=\right.$ $\left.s_{j} \mid Z_{0}=s_{i}\right)$, which in turn can be used to calculate $\operatorname{Pr}\left(V_{t}=v, Z_{t}=s_{j} \mid Z_{0}=s_{i}\right)$ and then $\operatorname{Pr}\left(V_{t}=v \mid Z_{0}=s_{i}\right)$.

$\mathbf{V}(t, v)=\left[\operatorname{Pr}\left(V_{t}=v, Z_{t}=s_{j} \mid Z_{0}=s_{i}\right)\right]$ denotes the matrix that captures the probability of having to wait $v$ time units to enter subset $\mathcal{A}$, and of being in state $s_{j}$ conditional on starting in state $s_{i}$. Let $\mathbf{W}(t, v)=\left[\operatorname{Pr}\left(V_{t} \geq v, Z_{t}=s_{j} \mid Z_{0}=s_{i}\right)\right]$ capture the probability of waiting at least $v$ time units. For $v=0$ and $v=0.5$, the probability can be calculated using

$$
\mathbf{W}(t, 0)=\mathbf{P}^{t}
$$

and

$$
\mathbf{W}(t, 0.5)=\left(\begin{array}{cc}
\mathbf{0} & \mathbf{0} \\
\mathbf{0} & \mathbf{P}_{\mathcal{B}}^{t}
\end{array}\right) .
$$

These formulas capture the history of the process until time $t$, and can be interpreted like the initial conditions for the time spent in subset $\mathcal{A}$ given by equations (4) and (5). Equation (8) gives the distribution of $Z_{t}$ conditional on $Z_{0}=s_{i}$, as $\operatorname{Pr}\left(V_{t} \geq 0, Z_{t}=\right.$ $\left.s_{j} \mid Z_{0}=s_{i}\right)=\operatorname{Pr}\left(Z_{t}=s_{j} \mid Z_{0}=s_{i}\right)$. Equation (9) shows that for the time to entry to be at least 0.5 , it is necessary to start in a state in $\mathcal{B}$, and to stay in one of these states until 
$t$. Either starting in $\mathcal{A}$ or moving from $\mathcal{B}$ to $\mathcal{A}$ until $t$ means that the first entry already happened until $t$, and thus does not appear in (9).

For $v=1.5,2.5, \ldots$ the development of the process beyond $t$ has to be accounted for. The following formula can be used:

$$
\mathbf{W}(t, v)=\left[\left(\begin{array}{cc}
\mathbf{0} & \mathbf{0} \\
\mathbf{0} & \mathbf{P}_{\mathcal{B}}^{v-0.5}
\end{array}\right)\left(\begin{array}{cc}
\mathbf{0} & \mathbf{0} \\
\mathbf{0} & \mathbf{1}_{m_{\mathcal{B}}}
\end{array}\right)\right]^{T} \circ \mathbf{W}(t, 0.5)
$$

$\mathbf{1}_{m_{\mathcal{B}}}$ is a matrix of the same dimensions as $\mathbf{P}_{\mathcal{B}}$ with all entries equal to one, $T$ denotes the transpose, and o denotes the Hadamard product. The matrix on the right side of the Hadamard product is given by equation (9), and accounts for the development of the process until $t$. The term on the left side of the Hadamard product is a calculation of the probability of staying in a state in subset $\mathcal{B}$ for $v$ or more time units, conditional on starting from a state in subset $\mathcal{B}$; formally $\sum_{s_{k} \in \mathcal{B}} \operatorname{Pr}\left(Z_{t+v-0.5}=s_{k} \mid Z_{t}=s_{j}\right)$ for $s_{j} \in \mathcal{B}$. For $v=1,2, \ldots \mathbf{W}(t, v)$ will equal $\mathbf{W}(t, v+0.5)$ because the transitions between states are assumed to occur mid-interval.

$\mathbf{V}(t, v)$ is calculated as

$$
\mathbf{V}(t, v)=\mathbf{W}(t, v)-\mathbf{W}(t, v+1)
$$

for $v=0.5,1.5, \ldots$ and

$$
\mathbf{V}(t, 0)=\mathbf{W}(t, 0)-\mathbf{W}(t, 0.5)
$$

Finally, the probability $\operatorname{Pr}\left(V_{t}=v \mid Z_{0}=s_{i}\right)$ can be calculated from the row sums of $\mathbf{V}(t, v)$.

Note that the process may never visit the subset $\mathcal{A}$, such that $V=\infty$. This will usually happen when one or more of the states in $\mathcal{B}$ are absorbing. In this case, both $\operatorname{Pr}\left(V_{t} \geq v \mid Z_{0}=s_{i}\right)$ and $\operatorname{Pr}\left(V_{t}=v \mid Z_{0}=s_{i}\right)$ will be non-zero for arbitrarily large values of $v$. Similar issues occur for the recursive schemes of Skoog and Ciecka (2010). If the Markov chain is absorbing, as is assumed here, then for $t$ and $v$ above some thresholds $t^{\prime}$ and $v^{\prime}$ only entries of $\mathbf{V}\left(t^{\prime}, v^{\prime}\right)$ are non-zero for which $s_{j}$ denotes an absorbing state, and the calculation can be stopped. Alternatively, or if the chain is not absorbing, a pre-specified time $t^{\prime \prime}$ may be used. If the researcher is interested in the distribution of $V$ conditional on $V$ being finite, it suffices to condition on $V<v^{\prime}$.

\subsection{Waiting time to final exit}

So far, Skoog and Ciecka (2010) are the only scholars who have presented an approach for calculating the time to last exit. While they used a Markov chain restricted to two non-absorbing states, the method presented below works with an arbitrary state space. The basic reasoning is again different from that of the two previous cases. For the distribution of occupation times, the history of the process has to be considered; and for the time to first entry, both the history of the process and its future development have to be taken into account. On the other hand, for the time to final exit from a subset $\mathcal{A}$, only the future development is of importance. Essentially, starting from $t=0$, the probability that the waiting time to final exit will equal $t+0.5$ is captured by the probability of being 
in subset $\mathcal{A}$ at time $t$, of being in $\mathcal{B}$ at $t+1$, and of staying in $\mathcal{B}$ thereafter. The exact path to being in $\mathcal{A}$ at $t$ is of no importance; i.e., it does not matter how the process ends up in $\mathcal{A}$. This means that up to $t$, the process can repeatedly move between $\mathcal{A}$ and $\mathcal{B}$.

Let $\mathbf{E}(t, e)$ denote the matrix with entries $\operatorname{Pr}\left(E_{t}=e, Z_{t}=s_{j} \mid Z_{0}=s_{i}\right)$, which capture the probability of exiting $\mathcal{A}$ for the last time in $e$ time units, and of being in state $s_{j}$ conditional on starting in state $s_{i}$. The distribution of the time to last exit, $\operatorname{Pr}\left(E_{t}=e \mid Z_{0}=s_{i}\right)$, can be calculated from the row sums of $\mathbf{E}(t, e)$.

To calculate $\mathbf{E}(t, e)$, it is assumed that transitions from $\mathcal{A}$ to $\mathcal{B}$ are only possible up to some time step $t=\tau_{E}+1$ of the process. This means that the last transition from $\mathcal{A}$ to $\mathcal{B}$ can occur mid-interval between $\tau_{E}$ and $\tau_{E}+1$; i.e., after the process has progressed $\tau_{E}+0.5$ time steps, and not any later. For some non-absorbing Markov chains, $\tau_{E}$ might not exist, and transitions between $\mathcal{A}$ and $\mathcal{B}$ are always possible. In this case, the researcher still needs to set a value for $\tau_{E}$ to apply the method described below. This changes the interpretation of the results slightly, as the additional qualification is needed that they are conditional on the final exit happening up to $\tau_{E}$ time steps, or earlier.

Assuming that there is some finite value for $\tau_{E}, \mathbf{E}\left(\tau_{E}, e\right)$ is given by

$$
\mathbf{E}\left(\tau_{E}, e\right)=\mathbf{0} \text { for } e \geq 1 \text {, }
$$

which formalizes the assumption stated above that no transitions occur after $\tau_{E}$, as at $t=\tau_{E}$ time to last exit can only equal zero or 0.5 . The corresponding probabilities are given by

$$
\mathbf{E}\left(\tau_{E}, 0\right)=\left[\left(\begin{array}{ll}
\mathbf{P}_{\mathcal{A}} & \mathbf{0} \\
\mathbf{P}_{\mathcal{B A}} & \mathbf{P}_{\mathcal{B}}
\end{array}\right) \mathbf{1}_{m}\right]^{T} \circ \mathbf{P}^{\tau_{E}}
$$

and

$$
\mathbf{E}\left(\tau_{E}, 0.5\right)=\left[\left(\begin{array}{ll}
\mathbf{0} & \mathbf{P}_{\mathcal{A B}} \\
\mathbf{0} & \mathbf{0}
\end{array}\right)\left(\begin{array}{cc}
\mathbf{0} & \mathbf{0} \\
\mathbf{0} & \mathbf{1}_{m_{\mathcal{B}}}
\end{array}\right)\right]^{T} \circ \mathbf{P}^{\tau_{E}}
$$

The matrix on the right side of the Hadamard product in both equation (14) and equation (15) keeps track of the state $s_{i}$ in which the process is at time $\tau_{E}$. In equation (14), the term on the left side of the Hadamard product captures whether there is no transition from $\mathcal{A}$ to $\mathcal{B}$. The term in equation (15) follows a similar logic, and accounts for transitions from $\mathcal{A}$ to $\mathcal{B}$.

For more general results, the following notation can be used:

$$
\mathbf{P}_{E}=\left(\begin{array}{ll}
\mathbf{0} & \mathbf{P}_{\mathcal{A B}} \\
\mathbf{0} & \mathbf{0}
\end{array}\right) \quad \text { and } \quad \mathbf{P}_{S}=\left(\begin{array}{ll}
\mathbf{P}_{\mathcal{A}} & \mathbf{0} \\
\mathbf{P}_{\mathcal{B} \mathcal{A}} & \mathbf{P}_{\mathcal{B}}
\end{array}\right)
$$

For $e=0.5,1.5, \ldots \mathbf{E}(t, e)$ can be written as

$$
\mathbf{E}(t, e)=\left(\mathbf{P}^{e-0.5} \mathbf{P}_{E} \mathbf{P}_{S}^{\tau_{E}-t-e-0.5} \mathbf{1}_{m}\right)^{T} \circ \mathbf{P}^{t}
$$

The matrix on the right side of the Hadamard product again gives the state at time $t$. The term on the left side, read from left to right, captures the future progress of the process by keeping track of the state the process is in until $t=e-0.5$. Then, a transition from 
$\mathcal{A}$ to $\mathcal{B}$ must occur for $E$ to equal $e$, as captured by $\mathbf{P}_{E} . \mathbf{P}_{S}^{\tau_{E}-t-e-0.5}$ accounts for the requirement of staying in $\mathcal{B}$ after moving there from $\mathcal{A}$. Finally, the identity matrix sums over all of the states the process can be in at time $\tau_{E}+1$, as only $Z_{t}$ and $Z_{0}$ are of interest. Note that

$$
\mathbf{E}(t, e)=\mathbf{0} \quad \text { for } \quad e \geq \tau_{e}-t+1 \text {. }
$$

For $e=0$

$$
\mathbf{E}(t, 0)=\left(\mathbf{P}_{S}^{\tau_{E}-t+1} \mathbf{1}_{m}\right)^{T} \circ \mathbf{P}^{t}
$$

can be used, and for $e=1,2, \ldots$

$$
\mathbf{E}(t, e)=\mathbf{0}
$$

Note that $E=0$ has to be interpreted differently than $V=0 . V=0$ means that the first entry already has occurred. If the first entry has not yet occurred and will not occur in the future $V=\infty$. By contrast, $E=0$ means that the last exit either has already occurred or will never occur.

For the last exit, it may be desirable to not just specify which subset is left, but also to specify whether the exit is directed to a specific subset. As before, $\mathcal{A}$ denotes the subset that is left. Let $\mathcal{B}$ denote the exit subset, and let $C$ denote the subset of all of the remaining states of $\mathcal{S}$ that are not of special interest. $\mathbf{P}_{E}$ and $\mathbf{P}_{S}$ can now be written as

$$
\mathbf{P}_{E}=\left(\begin{array}{lll}
\mathbf{0} & \mathbf{P}_{\mathcal{A B}} & \mathbf{0} \\
\mathbf{0} & \mathbf{0} & \mathbf{0} \\
\mathbf{0} & \mathbf{0} & \mathbf{0}
\end{array}\right) \quad \text { and } \quad \mathbf{P}_{S}=\left(\begin{array}{lll}
\mathbf{P}_{\mathcal{A}} & \mathbf{0} & \mathbf{P}_{\mathcal{A C}} \\
\mathbf{P}_{\mathcal{B} \mathcal{A}} & \mathbf{P}_{\mathcal{B}} & \mathbf{P}_{\mathcal{B C}} \\
\mathbf{P}_{C \mathcal{A}} & \mathbf{P}_{C \mathcal{B}} & \mathbf{P}_{C}
\end{array}\right)
$$

Otherwise, the same formulas as before can be used, but they now give the time to last exit to subset $\mathcal{B}$.

\section{Application to working life tables for Spanish males}

\subsection{Background}

In 2008, the Spanish economy was hit hard by the global financial crisis that has since been aptly named the "Great Recession". According to Eurostat, the unemployment rate in Spain increased dramatically, from $8.2 \%$ in 2007 to $24.8 \%$ in 2012, and it remains high (19.6\% in 2016). Several explanations for the severe impact of the crisis on Spain have been proposed, including reasons related to the structure of the Spanish labor market, which is segregated into two groups: highly protected workers with permanent contracts and individuals with weakly protected fixed-term contracts (Bentolila et al., 2012a,b). Moreover, the Spanish labor market is characterized by high levels of overqualification and by regional mismatches between labor demand and supply (Dolado et al., 2013). These factors imply that the effects of the recession may have been heterogeneous, and that individuals who were well integrated in the labor market and were protected by permanent contracts might not have been as affected as individuals who were already in a more precarious position before the recession. 
While there is evidence that the length of working life decreased due to the recession, and that the impact of the crisis was indeed heterogeneous (Dudel et al., 2016), it is less clear to what degree the observed losses in working life expectancy are due to a compression of working life (i.e., to later entry into and/or earlier exit from working life), or to working life consisting of more and longer periods of temporary withdrawal from the labor force. Earlier research has provided evidence for both effects.

First, it appears that young Spaniards have been entering the labor market later in an effort to avoid becoming unemployed, as unemployment rates in Spain are especially high among young adults (Dolado et al., 2013). The evidence regarding earlier exits is less clear. In the past, individuals who exited the labor market early often went through disability programs rather than old-age pension schemes, as disability programs were less restrictive, and the disability benefits tended to be more generous than the pension benefits available to early retirees (García-Gómez et al., 2012; Benavides et al., 2015). However, since recent reforms have made this pathway less attractive, fewer Spaniards are collecting disability benefits (Silva and Vall-Castello, 2012). Nonetheless, displaced workers in Spain still have a high probability of retiring after reaching age 60 (Tatsiramos, 2010), and these early labor market exits may be associated with the recession.

Second, there is also clear evidence of increases in the average number and length of spells of temporary withdrawal from the labor force (Dudel et al., 2016). This pattern has been observed among individuals who have become unemployed, are not entitled to unemployment benefits, and do not expect to find a job (Congregado et al., 2011). These individuals leave the labor market as so-called "discouraged workers", although they might return to the labor market when economic conditions improve. Moreover, unemployed individuals may lose their eligibility for unemployment benefits after a period of time (Venn, 2012). These workers might then become discouraged workers as well, or they might find work in informal labor.

\subsection{Data}

The data comes from the Continous Working Life Sample (CWLS; Muestra Continua de Vida Laboral) for the years from 2004 to 2013. The CWLS is a 4\% random sample from the Spanish social security register, which covers all individuals who are either making contributions to the social security system or are receiving social security benefits (López Gómez et al., 2016).

The initial sample was taken in 2004, and sample members are followed over time. Individuals who lose contact with the social security as a consequence of becoming inactive drop out of the sample, and are replaced with new individuals randomly drawn from the register; however, these individuals might reenter the sample if they resume or start receiving benefits or making contributions. As the dataset does not include inactive persons, it is selective with respect to this labor force state. A similar problem exists for migration out of Spain: individuals who leave the country are lost to the sample, but may reenter. While it has been shown that this could bias results for females, results for males are affected to a lesser extent (Dudel et al., 2016). For this reason, the working life tables are reported for males only, corrected for the selectivity of the dataset (see below). The date of death is also recorded in the CWLS. As the estimates of mortality rates and life expectancy derived from the CWLS are closely aligned with the life tables of the 
Human Mortality Database (2015), it is assumed that the dates of death are recorded correctly.

In total, the working life trajectories of 781, 599 males are included in the analyses. For each year and each individual, a labor force state was generated based on which state the individual occupied for the majority of the year. The states shown in figure 1 ("active", "inactive", "retired", "disabled", and "dead") combined with ages 15 to 99 are considered. It is assumed that everyone aged 15 and under is inactive. Due to the nature of the dataset, the states are based on paying contributions or receiving benefits: active individuals either make social security contributions or receive unemployment benefits; retirement is defined through the receipt of pension benefits; and individuals count as disabled if they receive disability benefits. If individuals are missing from the dataset for one or more years, it is assumed that they are inactive, provided that they reenter the dataset later or that no date of death is given.

\subsection{Estimation}

Over the period from 2004 to 2013, there are 6,622, 086 transitions in the dataset; i.e., transitions from being in state $s_{i}$ in year $t$ to being in state $s_{j}$ in year $t+1$. The transitions are modeled using discrete event history analysis (e.g., Allison, 1982). The transition probabilities are estimated using multinomial logistic regression using the state at time $t+1$ as the dependent variable, which leads to four equations. The state at time $t$ is used as one of the explanatory variables. Period is controlled for by including dummy variables. Age is included using cubic smoothing splines (Yee and Wild, 1996). This regression model is applied to each of five subsamples. The first subsample covers ages 15 to 29 (early working life); the second subsample includes ages 30 to 54 (prime working age); the third subsample covers ages 55 to 64 (transition to retirement); the fourth subsample starts with age 65 and includes all ages up to 79; and the fifth subsamples covers ages 80 to 99 (old age). Our decision to perform the estimation by subsamples was motivated by computational issues caused by the large sample size, but an additional benefit of this approach is that it implicitly introduces interactions with all variables by age group, and allows for discontinuities in the otherwise smooth age trajectory. The transition probabilities resulting from this estimation exercise were adjusted using the non-parametric correction procedure proposed by Dudel et al. (2016), which relies on external data sets and corrects for the selectivity of the CWLS.

The transition probabilities estimated from transitions between 2004 and 2005 are used for a Markov chain, viz., a working life table; the transitions between 2005 and 2006 are used for another Markov chain, and so on. The indicators derived for each working life table thus follow a period perspective, and show how individuals would fare if the conditions of each pair of years (e.g., 2004/2005) prevailed for a period spanning the greater part of life starting from age 15 . The use of this period perspective is standard practice for the estimation of working life tables (e.g., Butt et al., 2008; Warner et al., 2010; Skoog and Ciecka, 2010).

In the current analysis, all of the periods up to 2006/2007 are considered as prerecession periods. The period 2007/2008 is considered to be a transition period because 2007 was not fully affected by the recession, whereas 2008 was fully affected. 2008/2009 and the following periods are considered as recession periods. To assess the effects of the 
recession,we compare the last pre-recession period 2006/2007 with the first recession period 2008/2009. We cannot rule out the possibility that factors other than the recession, such as policy changes, had an impact on the indicators that are calculated. Still, the recession itself can be seen as an exogenous shock for the labor market. Given that before and after this shock levels of year-to-year variation in the labor market were comparatively low, it is plausible to assume that the recession was the main driver of the differences between 2006/2007 and 2008/2009.

For each of the periods, the expected lifetime spent in each of the labor force states ("active", "inactive", "retired", "disabled") starting from age 15 and being inactive is calculated. Age 15 and being inactive is also used as the conditioning state for all of the other results, as in Spain individuals are not allowed to work before age 16. Thus, this state is a natural starting point. The results should be interpreted accordingly. For instance, if working life expectancy is reported to be $x$ years, this means that an inactive 15 year old Spanish male could expect to have $x$ years of labor market activity, given that the conditions of a specific period prevail, as defined above.

Using the methods described in this paper, the quartiles of time spent in each state are calculated. This allows us to assess the heterogeneity of the effects of the recession. The interquartile range is used to assess whether inequalities in the duration of working life have been affected by the recession. Moreover, the age at first entry into the labor force and the age at final exit are estimated, where the final exit can be through any of the other states. It is important to distinguish the latter age from the age at retirement, which only captures one of the three possible pathways out of the labor market. For instance, disability was - and to some degree still is - a form of early retirement in Spain (García-Gómez et al., 2012; Benavides et al., 2015). Combining all of these measures will allow us to draw a more complete picture of the impact of the recession on working life in Spain. ${ }^{3}$

For all of the calculations, the free statistical software $\mathrm{R}$ was employed (R Core Team, 2015). The vgam package for $\mathrm{R}$ was also used (Yee, 2010). All of the code and the (intermediate) results are available online.

\subsection{Results}

Figure 2 shows how the expected lifetime spent in each of the four states has developed over time. The time spent in disability and the time spent in retirement do not seem to have been affected by the recession: the time spent in disability has fluctuated at around two years, while the time spent in retirement has increased slowly but steadily in lockstep with increases in life expectancy. In contrast, there have been drastic changes in working life expectancy (WLE; active state): WLE was 39.7 years in 2006/2007, and fell 6.4 years to 33.3 years in 2008/2009. The lifetime spent in the inactive state is the mirror image of WLE, and increased by 6.6 years. After the 2008/2009 period, both WLE and the lifetime spent in the inactive state fluctuated somewhat, but roughly stayed at the same level.

\footnotetext{
${ }^{3}$ While standard errors and related quantities are not of major interest here due to the large sample size of the data, they can be calculated using the bootstrap for all quantities presented in this paper (Craig and Sendi, 2002).
} 


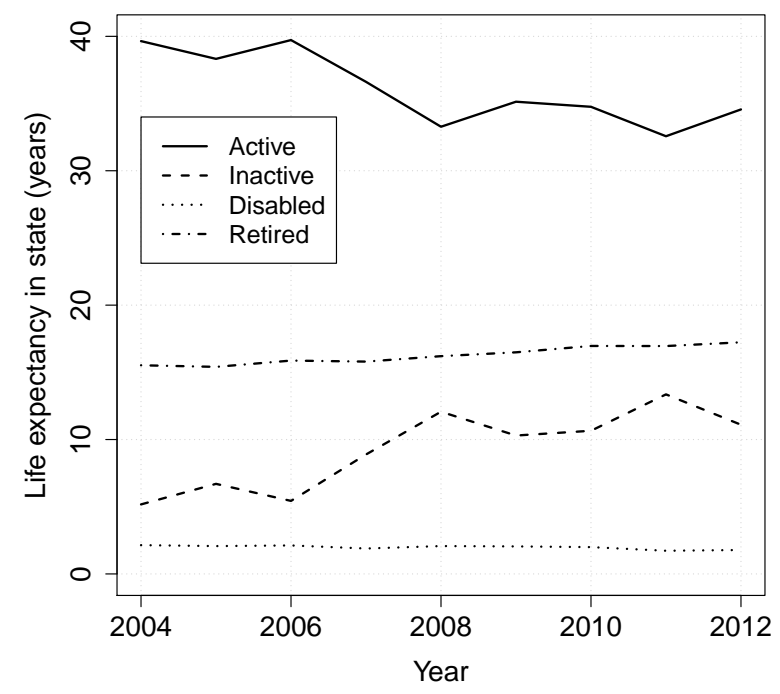

Figure 2: Life expectancy of Spanish males spent in labor market activity, inactivity, disability, and retirement (measured in years); 2004-2013.

While results like those from figure 2 are routinely calculated in the literature, the findings shown in figures $3 \mathrm{a}$ and $3 \mathrm{~b}$ go beyond the results that are usually presented. These figures depict the quartiles of the distribution of WLE (fig. 3a) and of the lifetime spent in the inactive state (fig. 3b), in addition to the respective expectation that was already shown in figure 2. As can be seen, the effect of the recession differs strongly by quartile. For WLE, the first quartile decreased by eight years and the median decreased by seven years, while the third quartile decreased by only five years. For the lifetime spent in inactivity, the changes are of the same magnitude, but have the opposite sign: the first quartile increased by four years, the median increased by six years, and the third quartile increased by 10 years. Taken together, these findings imply that those individuals who had low labor market attachment before the recession lost more WLE than those who stayed long in the labor market. Moreover, the interquartile range, and thus the levels of inequality in WLE and of the lifetime spent in inactivity, increased: for WLE, the interquartile range was nine years pre-recession, and increased by three years to 12 years during the recession. For the lifetime spent in inactivity, the interquartile range was four years pre-recession and 10 years during the recession.

None of the occupation times presented so far necessarily imply a consecutive sequence of years. For example, at one extreme, the WLE estimate of 39.7 years in 2006/2007 could mean a consecutive sequence of 39.7 years, but it could also reflect any number of episodes of activity with breaks in-between that add up to 39.7 years of activity. This makes the drop in working life expectancy of 6.4 years in 2008/2009 hard to interpret: did the period in which individuals occupy the "active" state become shorter due to later entry and earlier exit, or did the breaks between activity episodes become longer or more common, or both? Figure 4 shows the results of an analysis based on the approaches presented in section 4 that allow us to disentangle this problem. Figure $4 \mathrm{a}$ shows the age at which the active state is reached for the first time, if it is reached at all, 


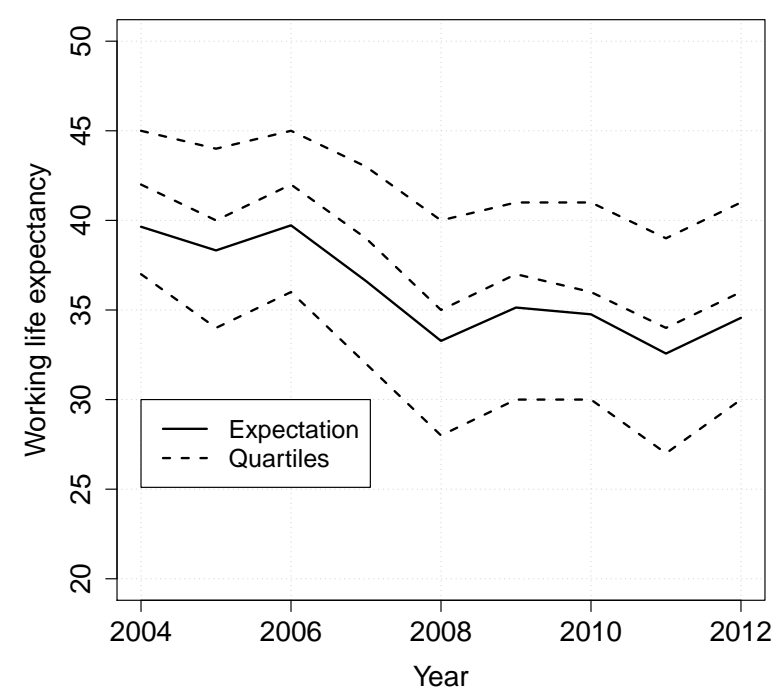

(a)

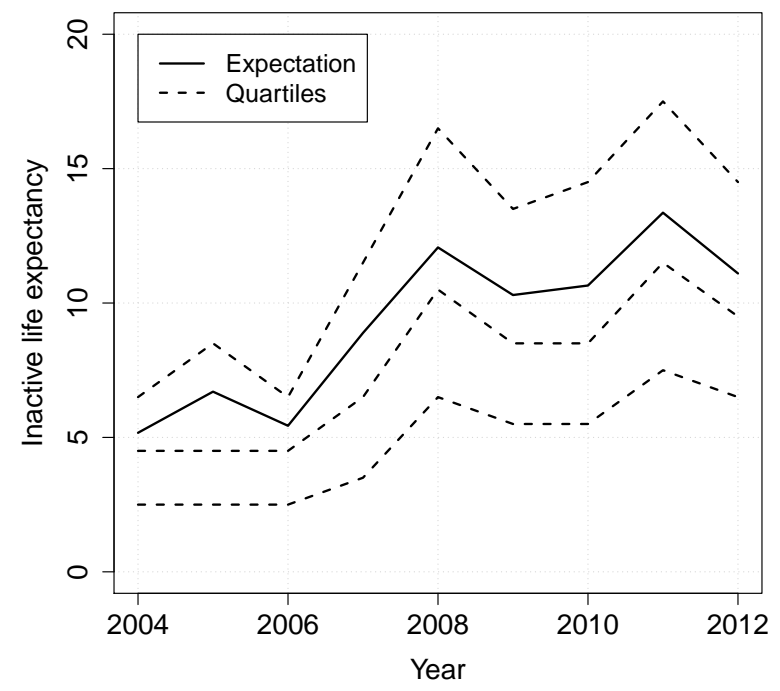

(b)

Figure 3: Quartiles (1st, 2nd, 3rd) of lifetime spend in activity (a) and inactivity (b). 


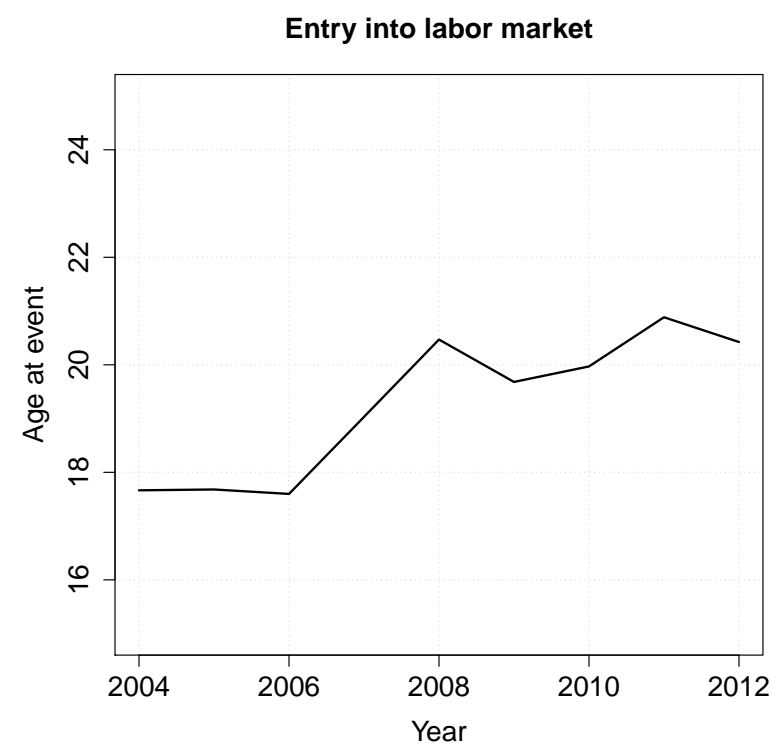

(a)

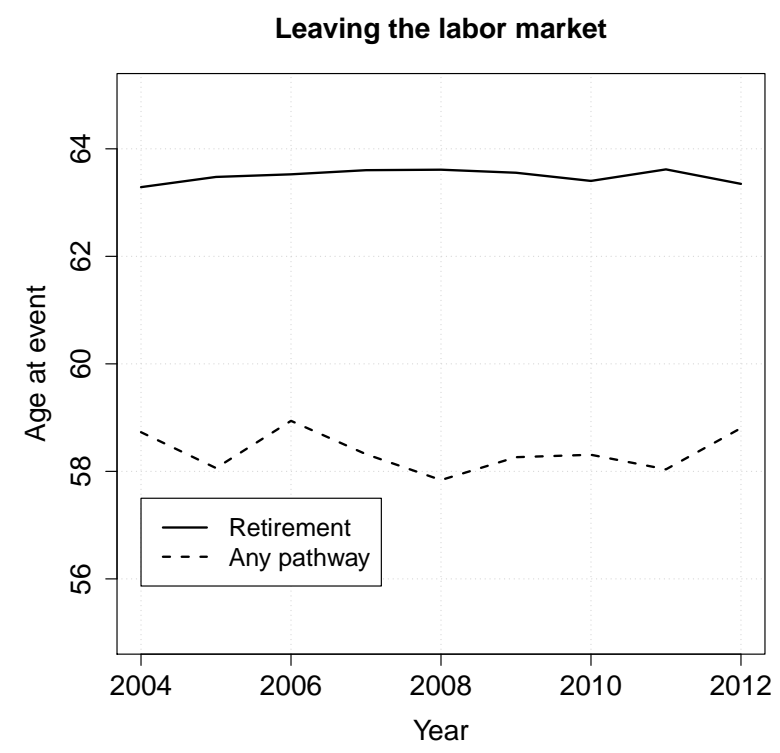

(b)

Figure 4: Age at first entry into the labor market (a) and age at leaving the labor market (b) defined as either the uptake of an old-age pension or the final exit from labor market activity. 
as is the case for the large majority of males. In figure $4 \mathrm{~b}$, the age at which labor force activity is left for the last time is shown; i.e., the age at final exit through retirement (solid line) and through any pathway, including, but not restricted to, retirement, and excluding death (dashed line).

The average age at first entry was around 17.6 years before the recession. It increased by roughly 2.9 years to around 20.5 years during the recession, which implies that young Spaniards delayed their entry into the labor market considerably. On the other hand, the age at withdrawal from the labor market did not change as much, and contributed to a lesser extent to the decrease in WLE. Measured as the average age at retirement (i.e., the average age at which individuals started receiving pension benefits), the age fluctuated only slightly at around 63.5 years. If measured as the final exit from the labor market through any pathway not restricted to retirement, the average age is about five years lower. Due to the recession, the age declined slightly from 58.9 years to 57.8 years. Taken together, these findings imply that roughly four years of the 6.4-year decrease in WLE can be explained by later entry to and earlier exit from the labor market.

\section{Conclusion}

In this paper, new methods for Markov chains were presented. These methods include a method for calculating the distribution of the time spend in a subset of the state space, a method for calculating the distribution of the waiting time until the first entry into a subset of states, and a method for calculating the distribution of the waiting time until the final exit from a subset of states. To provide an example of how they can be used, the methods were applied to working life tables for Spain in order to assess the impact of the financial crisis on the length of working life. The recession that followed the financial crisis of 2007/2008 had a tremendous impact on the Spanish labor force, leading to a decrease in working life expectancy of more than six years. By applying the methods described in this paper, we demonstrated that around $60 \%$ of this decrease could be explained by later entry into and earlier exit from the labor market. Moreover, we found that the impact of the recession was rather heterogeneous, and that individuals who already had a relatively low working life expectancy before the recession experienced greater decreases than individuals who had a relatively high working life expectancy; these effects led to an increase in the levels of inequality in the length of working life.

These insights would not have been possible using the standard Markov chain tools, and the example highlights the usefulness of the methods presented here, which provide deeper insights into the process under study, and yield easily interpretable quantifications of its underlying dynamics. Still, whether an application of these methods yields valid results depends on the validity of the discrete time, discrete state Markov chain model for a given application, and on valid estimates of transition probabilities. For instance, Langeheine and van de Pol (1990) have pointed out two commonly occurring reasons why the Markov model might be inappropriate: measurement error and population heterogeneity. Both issues have been addressed in the literature (e.g., Heckman and Singer, 1982; Gill, 1992; Liu et al., 1997; van den Hout et al., 2014). While these works show that potential issues can be tackled, the methods presented in this paper do not 
resolve them. It might therefore be necessary to use these methods in conjunction with other approaches.

The application presented in this paper focused on working life tables commonly used by sociologists, demographers, economists, and actuaries; but the methods developed here are not limited to this example. Other potential applications include, but are not limited to, multistate life tables modeling family states (e.g., first entry into marriage; final exit from being single), migration (e.g., first move; final return to home region), and health (e.g., first period of disability; final exit from healthy to sickness).

\section{References}

Allison, P. D. (1982), "Discrete-Time Methods for the Analysis of Event Histories," Sociological Methodology 13: 61-98.

Benavides, F. G., Durán, X., Gimeno, D., Vanroelen, C., and Martínez-Martínez, J. M. (2015), "Labour market trajectories and early retirement due to permanent disability: A study based on 14972 new cases in Spain.” European Journal of Public Health 25: 673-677.

Bentolila, S., Cahuc, P., Dolado, J. J., and Barbanchon, T. L. (2012a), “Two-Tier Labour Markets in the Great Recession: France versus Spain," Economic Journal 122: F155F187.

Bentolila, S., Dolado, J. J., and Jimeno, J. F. (2012b), "Reforming an insider-outsider labor market: The Spanish experience," IZA Journal of European Labor Studies 1: $1-29$.

Butt, Z., Haberman, S., and Verral, R. (2008), "Calculating compensation for loss of future earnings: Estimating and using working life expectancy," Journal of the Royal Statistical Society, Series A 171: 763-805.

Caswell, H. (2001), Matrix Population Models, Sinauer.

Congregado, E., Golpe, A. A., and van Stel, A. (2011), "Exploring the big jump in the Spanish unemployment rate: Evidence on an 'added-worker' effect," Economic Modelling 28: 1099-1105.

Craig, B. A. and Sendi, P. P. (2002), "Estimation of the transition matrix of a discretetime Markov chain," Health Economics 11: 33-42.

Crimmins, E. M., Hayward, M. D., and Saito, Y. (1996), "Differentials in Active Life Expectancy in the Older Population of the United States," Journal of Gerontology: Series B 51B: S111-S120.

Dolado, J. J., Jansen, M., Felgueroso, F., Fuentes, A., and Wölfl, A. (2013), "Youth Labour Market Performance in Spain and its Determinants: A Micro-Level Perspective," OECD Economics Department Working Papers 1039.

Dudel, C., López Gómez, M., Benavides, F., and Myrskylä, M. (2016), "A lost generation? The financial crisis and the length of working life in Spain," MPIDR Working Paper WP-2016-010.

García-Gómez, P., Jiménez-Martín, S., and Castelló, J. V. (2012), "Health, Disability, and Pathways into Retirement in Spain," in Social Security Programs and Retirement around the World, ed. Wise, D. A., Chicago: University of Chicago Press, pp. 127174. 
Gill, R. D. (1992), "Multistate life table and regression models," Mathematical Population Studies 3: 259-276.

Grinstead, C. M. and Snell, J. L. (1997), Introduction to Probability, American Mathematical Society.

Haberman, S. and Bloomfield, D. S. F. (1990), "Work Time Lost to Sickness, Unemployment and Stoppages: Measurement and Application," Journal of the Institute of Actuaries 117: 533-595.

Hayward, M. D., Friedman, S., and Chen, H. (1996), "Race Inequities in Men's Retirement," Journal of Gerontology: Social Sciences 51B: S1-S10.

Hayward, M. D. and Grady, W. R. (1990), "Work and Retirement Among a Cohort of Older Men in the United States, 1966-1983," Demography 27: 337-356.

Hayward, M. D. and Richter, D. L. (1998), "A Life Cycle Model of Labor Force Inequality," Sociological Methods and Research 26: 487-510.

Heckman, J. J. and Singer, B. (1982), "Population Heterogeneity in Demographic Models," in Multidimensional Mathematical Demography, eds. Land, K. C. and Rogers, A., New York: Academic Press, pp. 567-599.

Hodge, R. W. (1966), "Occupational mobility as a probability process," Demography 3: 19-34.

Hoem, J. M. (1977), “A Markov Chain Model of Working Life Tables," Scandinavian Actuarial Journal 1977: 1-20.

Human Mortality Database (2015), "University of California, Berkeley (USA), and Max Planck Institute for Demographic Research (Germany)," available at www.mortality.org or www.humanmortality.de. Accessed 30 July 2016.

Iosifescu, M. (1980), Finite Markov Processes and Their Applications, Dover.

Jimeno, J. F. and Santos, T. (2014), "The crisis of the Spanish economy," Journal of the Spanish Economic Association 5: 125-141.

Karas Montez, J. and Hayward, M. D. (2014), "Cumulative Childhood Adversity, Educational Attainment, and Active Life Expectancy Among U.S. Adults," Demography 51: 413-435.

Kemeny, J. G. and Snell, J. L. (1971), Finite Markov Chains, Springer.

Langeheine, R. and van de Pol, F. (1990), "A Unifying Framework for Markov Modeling in Discrete Space and Discrete Time," Sociological Methods and Research 18: 416441.

Leinonen, T., Martikainen, P., and Myrskylä, M. (2016), "Working life and retirement expectancies at age 50 by social class: Period and cohort trends and projections for Finland," Journal of Gerontology, Series B: Psychological Sciences and Social Sciences, Advance acess.

Liefbroer, A. C. and Henkens, K. (1999), "Labour market careers of successive cohorts of older men in the Netherlands: Changes in age at retirement and in length of working lives," Genus 55: 101-119.

Liu, X., Liang, J., Tu, E., and Whitelaw, N. (1997), "Modeling Multidimensional Transitions in Health Care," Sociological Methods and Research 25: 284-317.

Loichinger, E. and Weber, D. (2016), "Trends in Working Life Expectancy in Europe," Journal of Aging and Health 28: 1194-1213. 
López Gómez, M. A., Durán, X., Zaballa, E., Sanchez-Niubo, A., Delclos, G. L., and Benavides, F. G. (2016), "Cohort profile: the Spanish WORKing life Social Security (WORKss) cohort study," BMJ Open, 6, e008555.

Lubitz, J., Cai, L., Kramarow, E., and Lentzner, H. (2003), "Health, Life Expectancy, and Health Care Spending among the Elderly," New England Journal of Medicine 349: 1048-1055.

Millimet, D. L., Nieswiadomy, M., Ryu, H., and Slottje, D. (2003), "Estimating worklife expectancy: An econometric approach," Journal of Econometrics 113: 83-113.

Millimet, D. L., Nieswiadomy, M., and Slottje, D. (2010), "Detailed estimation of worklife expectancy for the measurement of human capital: Accounting for marriage and for children," Journal of Economic Surveys 24: 339-361.

Nurminen, M. M., Heathcote, C. R., Davis, B. A., and Puza, B. D. (2005), "Working life expectancies: The case of Finland 1980-2006," Journal of the Royal Statistical Society, Series A 168: 567-581.

Prais, S. J. (1955), "Measuring Social Mobility,” Journal of the Royal Statistical Society, Series A 118: 56-66.

R Core Team (2015), "R: A Language and Environment for Statistical Computing," Vienna, Austria.

Rogers, A. (1975), Introduction to Multiregional Mathematical Demography, New York: Wiley.

Rogers, A. and Ledent, J. (1976), "Increment-Decrement Life Tables: A Comment," Demography 13: 287-290.

Rogers, A., Rogers, R. G., and Belanger, A. (1990), "Longer Life but Worse Health? Measurement and Dynamics," The Gerontologist 30: 640-649.

Schmertmann, C. P., Amankwaa, A. A., and Long, R. D. (1998), "Three strikes and you're out: Demographic analysis of mandatory prison sentencing," Demography 35: 445-463.

Schoen, R. (2016), "Multistate Transfer Rate Estimation from Adjacent Populations," Population Research and Policy Review 35: 217-240.

Schoen, R., Landale, N. S., and Daniels, K. (2007), "Family transitions in Young Adulthood," Demography 44: 807-820.

Sericola, B. (2000), "Occupation times in Markov processes," Stochastic Models 16: 479-510.

Silva, J. I. and Vall-Castello, J. (2012), "Evaluating the Impact of a Reduction in the Generosity of Disability Benefits: The 2008 Spanish Reform," IZA Discussion Paper 6482.

Skoog, G. R. and Ciecka, J. E. (2002), "Probability mass functions for additional years of labor market activity induced by the Markov (increment-decrement) model," Economics Letters 77: 425-431.

- (2010), "Measuring Years of Inactivity, Years in Retirement, Time to Retirement, and Age at Retirement within the Markov Model," Demography 47: 609-628.

Smith, S. J. (1986), "Worklife Estimates: Effects of Race and Education,” Bureau of Labor Statistics Bulletin 2254.

Stander, J., Farrington, D. P., Hill, G., and Altham, P. M. E. (1989), "Markov Chain Analysis and Specialization in Criminal Careers," British Journal of Criminology 29: 
317-335.

Tatsiramos, K. (2010), "Job displacement and the transitions to re-employment and early retirement for non-employed older workers," European Economic Review 54: 517-535.

Taylor, H. M. and Karlin, S. (1984), An Introduction to Stochastic Modeling, Academic Press.

van den Hout, A. and Matthews, F. E. (2009), "Estimating stroke-free and total life expectancy in the presence of non-ignorable missing values," Journal of the Royal Statistical Society, Series A 173: 331-349.

van den Hout, A., Ogurtsova, E., Gampe, J., and Matthews, F. E. (2014), "Investigating healthy life expectancy using a multi-state model in the presence of missing data and misclassification," Demographic Research 30: 1219-1244.

Venn, D. (2012), "Eligibility Criteria for Unemployment Benefits," OECD Social, Employment and Migration Working Papers, No. 131.

Warner, D. F., Hayward, M. D., and Hardy, M. A. (2010), "The Retirement Life Course in America at the Dawn of the Twenty-First Century," Population Research and Policy Review 29: 893-919.

Willekens, F. (1980), "Multistate analysis: Tables of working life," Environment and Planning A 12: 563-588.

Willekens, F., Shah, I., Shah, J. M., and Ramachandran, P. (1982), "Multi-state analysis of marital status life tables: Theory and application," Population Studies 36: 129-144.

Wolf, D. A. and Gill, T. M. (2009), "Modeling transition rates using panel current-status data: How serious is the bias?" Demography 46: 371-386.

Yee, T. W. (2010), "The VGAM Package for Categorial Data Analysis," Journal of Statistical Software 32: 1-34.

Yee, T. W. and Wild, C. J. (1996), "Vector Generalized Additive Models," Journal of the Royal Statistical Society, Series B 58: 481-493. 